\title{
Mise Au Point Des Techniques De Micropropagation Pour Une Multiplication Massive De Gladiolus Segetum Et Gladiolus Dubius
}

\author{
Emna El Chaieb \\ Institut Supérieur Agronomique. Chott Mariem, Sousse, Tunisie \\ Faouzi Haouala \\ Institut National Agronomique de Tunisie, Tunis Belvédère, Tunisie
}

doi: 10.19044/esj.2016.v12n6p207 URL:http://dx.doi.org/10.19044/esj.2016.v12n6p207

\begin{abstract}
Apical buds of corms of gladiolus (Gladiolus segetum and Gladiolus dubius) were cultivated on Murashige and Skoog (1962) medium supplemented with IBA $0.1 \mathrm{mg} . \mathrm{l}^{-1}$ and BA $2 \mathrm{mg} . \mathrm{l}^{-1}$. The number of newly formed buds was 3,8 and 4,13 buds per explants for respectively Gladiolus segetum and Gladiolus dubius. The in vitro Rooting need a supplement with auxin and sucrose. Rooting is not present in the control medium. The rate of rooting varied from 10 to $36 \%$, depending on the species and the concentration of IBA in the medium. The Important rooting (36\%) is observed for Gladiolus segetum in the presence of IBA $1 \mathrm{mg} . \mathrm{l}^{-1}$. Finally, the acclimatization is possible, these shoots rooted have formed many corms after 6 weeks of culture.
\end{abstract}

Keywords: Gladiolus segetum, Gladiolus dubius, in vitro, multiplication, rooting.

\section{Résumé}

Les essais de multiplication in vitro ont montré que l'initiation de la culture est possible sur le milieu de Murashige et Skoog (1962) additionné d'AIB 0,1 mg. $\mathrm{l}^{-1}$ et BA $2 \mathrm{mg} . \mathrm{l}^{-1}$. La meilleure réponse est obtenue par les explants prélevés des bourgeons apicaux des bulbes. Ce milieu riche en BA, a permis d'avoir 3,83 et 4,1 bourgeons néoformés pour les explants prélevés des bourgeons apicaux. L'enracinement in vitro nécessite un milieu de culture MS additionné d'une auxine et de saccharose. L'enracinement est nul sur un milieu dépourvu d'hormone. Le taux d'enracinement de ces pousses atteint $36 \%$ en présence d'AIB $1 \mathrm{mg} . \mathrm{l}^{-1}$ chez Gladiolus segetum. Enfin, l'acclimatation est possible, Les pousses enracinées ont formé toutes des cormes après 6 semaines de culture. 
Mots-clés. Gladiolus segetum, Gladiolus dubius, in vitro, multiplication, enracinement.

\section{Introduction}

La flore tunisienne dispose de deux espèces sauvages de glaïeuls : Gladiolus italicus Mill. (G. segetum Ker-Gawl) et Gladiolus dubius qui couvrent certains domaines au milieu des champs et des prairies (Le Floc'h et al., 2010). Depuis 1970, la multiplication in vitro du glaïeul a été étudiée par plusieurs chercheurs (Hussey, 1977 ; Ziv, 1979 ; Steinitz et al., 1991 ; Bajaj et al., 1992 ; Bettaieb, 2003...).

La multiplication in vitro du Gladiolus a été réalisée à partir de bourgeons axillaires (Begum et Haddiuzaman, 1995; Boonvanno et Kanchanapoom, 2000, Salhi, 2007), d'apex (Hussain et al., 2001), de cormes (Nagaraju et Parthasarathy, 1995) et d'inflorescences (Ziv et Lilien-Kipnis, 2000). La multiplication in vitro est basée sur le développement de bourgeons axillaires préexistants après leur mise en culture sur un milieu contenant une cytokinine qui lève la dormance apicale et empêche la bulbification (Begum et Hadiuzzaman 1995; Grewal et al., 1995; Sen et Sen, 1995 ; Churvikova et Barykina, 1995).

Le déclenchement ou la stimulation du bourgeonnement axillaire en culture in vitro résulte de l'emploi de cytokinines, éventuellement associées aux auxines (Margara, 1984). La néoformation de bourgeons exige un rapport cytokinines/auxines élevé.

Tan Nhut et al. (2004) ont obtenu des bourgeonnements axillaires chez le glaïeul en utilisant la Benzyladénine (BA) à une gamme de concentrations comprises entre 2,2 et $4,5 \mu \mathrm{M}$. Dans une autre étude et après des essais de plusieurs combinaisons hormonales BA/AIB, le meilleur bourgeonnement a été obtenu avec un rapport BA/AIB égal à 4 (Bettaieb, 2003).

Pour l'enracinement et la bulbaison, l'utilisation d'une balance hormonale riche en auxine est souvent nécessaire. Le comportement d'un explant mis en milieu contenant un rapport auxine/cytokinine élevé évolue vers la rhizogenèse.

L'enracinement et la bulbaison in vitro sont obtenus après un séjour dans un premier milieu gélosé composé du milieu de Murashige et Skoog (MS) additionné d'AIB 0,5 mg.l . $^{-1}$ et 30 jours plus tard, dans le même milieu mais sans agar et riche en saccharose 6\% (Bettaieb, 2003). La micropropagation du glaïeul est possible à partir de bourgeons apicaux prélevés à partir de cormes (Haouala et Emna, 2012 ; Ascough, 2011 ; Goo et al., 2003; Priyakumari \& Sheela, 2005; Prasad \& Gupta, 2006; Roy et al., 2006; Aftab et al., 2008). L'initiation et la multiplication in vitro de cette 
plante sont réalisées sur un milieu de base MS additionné de BA 2 mg.l ${ }^{-1}$ et AIB 0,5 mg. $\mathrm{l}^{-1}$.

Dans ce chapitre, on s’est intéressé à la micropropagation de ces deux espèces. Pour cela, différents milieux de culture ont été testés afin d’avoir le meilleur taux de régénération. Le but de ce travail est la multiplication massive de ces deux espèces tout en préservant leurs caractéristiques génétiques en vue d’une éventuelle conservation.

\section{Matériel et méthodes}

\section{Matériel végétal}

Le matériel végétal utilisé est constitué de bourgeons apicaux prélevés sur les cormes adultes de deux espèces de glaïeul spontanées en Tunisie: Gladiolus segetum et Gladiolus dubius. Les cormes ont été récoltées au mois de juin 2012 à l’Institut supérieur agronomique de Chott Meriem, puis conservées au laboratoire des Sciences horticoles à la température ambiante. Une identification des espèces a été faite d'après les caractères morphologiques.

\section{Désinfection du matériel végétal}

Les bulbes sont nettoyés soigneusement par l'élimination des feuilles sèches, puis bien rincés à l'eau courante. Sous une hotte à flux laminaire, les bulbes sont trempés d'abord dans l'alcool à $70^{\circ}$ pendant quelques secondes puis dans une solution d'hypochlorite de sodium à $10 \%$ pendant $10 \mathrm{~min}$ et finalement dans une solution de $\mathrm{HgCl}_{2}$ à $0,1 \%$ pendant $5 \mathrm{~min}$. Les bulbes sont ensuite soumis à 3-4 rinçages successifs à l'eau distillée stérilisée.

\section{Milieux de culture et conditions expérimentales}

Le milieu de culture de base comprend les macroéléments, les microéléments et les éléments organiques de Murashige et Skoog (MS) (1962)

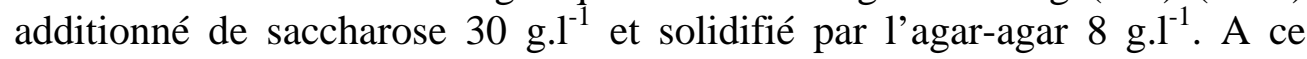
milieu de culture, différentes combinaisons hormonales sont ajoutées. Les hormones utilisées sont : Acide $\beta$-indole butyrique (AIB) et la benzyladénine (BA). Le pH des milieux de culture est ajusté à 5,8. Les cultures sont placées dans une chambre de culture climatisée sous un éclairement et une température contrôlés. La température est maintenue à $24 \pm 1^{\circ} \mathrm{C}$. L'éclairement est assuré par des tubes fluorescents assurant une intensité lumineuse de $35 \mu \mathrm{mol} . \mathrm{m}^{-2} . \mathrm{s}^{-1}$. La photopériode est de $16 \mathrm{~h}$ d'éclairement.

\section{Phase d'initiation}

Pour la phase d'initiation, le milieu de base a été additionné de différentes concentrations d'AIB et de BA:

- $\quad$ Milieu 1 : milieu MS sans hormones 
- $\quad$ Milieu 2 : milieu MS + BA 1 mg. $l^{-1}$

- $\quad$ Milieu 3 : milieu MS + AIB 0,1 mg. $.^{-1}+$ BA 1 mg.l ${ }^{-1}$

- $\quad$ Milieu 4 : milieu MS + AIB 0,1 mg. $.^{-1}+$ BA 2 mg. $l^{-1}$

Les milieux de culture sont stérilisés par autoclavage sous une pression de 1 bar et à une température de $120^{\circ} \mathrm{C}$. Le nombre de répétitions est de 12 tubes par milieu et type d'explant.

Les observations ont porté sur le nombre de bourgeons par explant et sur la longueur des pousses.

\section{Phase de multiplication}

Le milieu qui a donné le nombre de pousses le plus élevé pendant la première phase est le milieu 7 contenant de l'AIB $0,1 \mathrm{mg} . \mathrm{l}^{-1}$ et du BA 2 mg.l ${ }^{1}$. Ce milieu est choisi pour toutes les subcultures de cette phase.

Les pousses obtenues pendant la phase d'initiation ont été individualisées et mises dans des bocaux en verre de $500 \mathrm{ml}$ de volume à raison de 5 pousses par bocal. Le nombre total de pousses par explant et par espèce est de 50.

Les observations, faites après quatre semaines, ont porté sur le taux multiplication et sur la longueur des pousses obtenues.

\section{Phase d'enracinement}

Les pousses obtenues à l'issue de la phase précédente et âgées de 4 semaines ont été incubées sur le milieu MS additionnés d'AIB à différentes concentrations $\left(0 ; 0,1 ; 0,5\right.$ et $\left.1 \mathrm{mg}^{-1} \mathrm{l}^{-1}\right)$. Les mesures et observations, faites après 4 semaines de culture, ont porté sur le taux d'enracinement, le nombre et longueur des racines par pousse.

\section{Phase d'acclimatation}

Les pousses enracinées sont repiquées en caissettes en matière plastique couvertes par un film plastique transparent. Le substrat de culture est composé de tourbe et de perlite à proportions égales. Les caissettes sont placées à l'Institut supérieur agronomique de Chott Meriem. Les jeunes plantules sont maintenues à une température de $27 \pm 3^{\circ} \mathrm{C}$, une humidité relative de $80-85 \%$ et humectées tous les deux jours par pulvérisation. Cette phase d'acclimatation a duré 60 jours.

\section{Analyses statistiques}

L'analyse de la variance (ANOVA) a été effectuée pour toutes les données à l'aide du logiciel SPSS.16. Des différences statistiquement significatives entre les moyennes ont été déterminées en utilisant le test de Duncan à $\alpha=0,05$. 


\section{Résultats}

\section{Phase d'initiation}

Trois jours après le repiquage des explants, on observe le débourrement des bourgeons apicaux sur tous les milieux testés. Le taux de bourgeonnement atteint $100 \%$ après quatre semaines de culture (tableau 1 ). Pour le nombre de bourgeons néoformés par explant, le milieu le plus

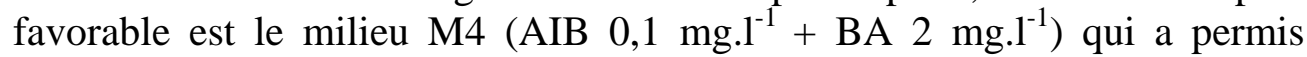
d'obtenir 3,8 et 4,1 bourgeons, respectivement chez Gladiolus segetum et Gladiolus dubius (figure 1). Pour tous les paramètres étudiés, l'analyse de la variance a montré que les différences entre les deux espèces ne sont pas significatives.

Tableau 1: Effets de l'AIB et de la BA sur l'initiation in vitro de bourgeons apicaux de Gladiolus segetum et de Gladiolus dubius.

\begin{tabular}{|c|c|c|c|c|}
\hline $\begin{array}{c}\text { Milieux } \\
\text { de culture }\end{array}$ & Espèces & $\begin{array}{r}\text { Taux de } \\
\text { bourgeonnement (\%) }\end{array}$ & $\begin{array}{c}\text { Nombre de bourgeons } \\
\text { néoformés } \\
\text { par explant }\end{array}$ & $\begin{array}{c}\text { Longueur } \\
\text { des pousses (cm) }\end{array}$ \\
\hline M1 & $\begin{array}{r}\text { G. segetum } \\
\text { G. dubius }\end{array}$ & 100 & $1 \pm 0$ bc & $0,55 \pm 0,15 \mathrm{~b}$ \\
M2 & G. segetum & 100 & $1 \pm 0$ bc & $0,62 \pm 0,22 \mathrm{~b}$ \\
\hline M3 & G. dubius & 100 & $1 \pm 0 \mathrm{bc}$ & $0,62 \pm 0,22 \mathrm{~b}$ \\
& G. segetum & 100 & $2,5 \pm 1 \mathrm{~b}$ & $0,74 \pm 0,51 \mathrm{~b}$ \\
\hline M4 dubius & 100 & $2,83 \pm 1,11 \mathrm{~b}$ & $0,78 \pm 0,51 \mathrm{~b}$ \\
& G. segetum & 100 & $1 \pm 0 \mathrm{bc}$ & $0,55 \pm 0,15 \mathrm{~b}$ \\
\hline
\end{tabular}

Les valeurs sur la même colonne portant des lettres distinctes sont significativement différentes $(\mathrm{P}<0,05)$.

Figure 1 : Elongation des bourgeons apicaux prélevés sur des cormes de Gladiolus segetum et cultivés sur le milieu M7 (AIB 0,1 mg. $.^{-1}+$ BA 2 mg. $l^{-1}$ ). 


\section{Phase de multiplication}

Les pousses axillaires obtenues à l'issue de la phase d'initiation ont été individualisées et repiquées sur le milieu d'initiation M4 (AIB 0,1 mg.l $\mathrm{l}^{-1}$

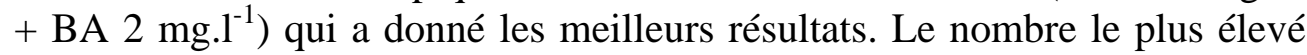
de bourgeons, est atteint, après quatre semaines de culture 4,2 et 4,3 bourgeons respectivement chez Gladiolus segetum et Gladiolus dubius (tableau 2). L'analyse de la variance montre une différence significative entre les 3 types d'explants, alors que cette différence non significative entre les deux espèces. La longueur des pousses varie de 1 à $1,5 \mathrm{~cm}$, toutefois, il n’y a pas de différences significatives entre les espèces (figures 2).

Tableau 2: Effet de la BA et de l'AIB sur le nombre de bourgeons et la longueur de pousses de deux espèces de genre Gladiolus cultivé in vitro.

\begin{tabular}{|c|c|c|}
\hline $\begin{array}{c}\text { Concentration des } \\
\text { hormones (mg. }{ }^{-1} \text { ) }\end{array}$ & Nombre de bourgeons & Longueur de pousses \\
\hline G. segetum & $4,20 \pm 1,99^{\mathrm{a}}$ & $1,48 \pm 1,47^{\mathrm{a}}$ \\
\hline G. dubius & $4,35 \pm 2,81^{\mathrm{a}}$ & $1,39 \pm 1,30^{\mathrm{a}}$ \\
\hline
\end{tabular}

Les valeurs sur la même colonne portant des mêmes lettres sont non significativement différentes $(\mathrm{P}<0,05)$.

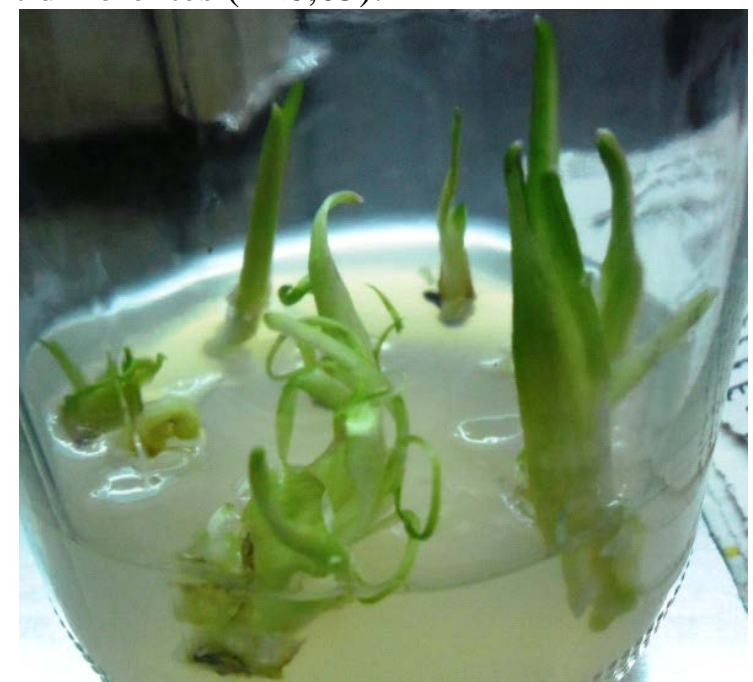

Figure 2 : Phase de multiplication des bourgeons apicaux sur le milieu M7 (AIB 0,1 mg. $\mathrm{l}^{-1}+$ BA 2 mg. ${ }^{-1}$ )

\section{Phase d'enracinement}

La phase d'enracinement a aboutit à une rhizogenèse des pousses à l'exception du milieu témoin (tableau 3). Le milieu le plus riche en auxine (AIB $1 \mathrm{mg} . \mathrm{l}^{-1}$ ) a donné le taux d'enracinement le plus élevé, soit 36 et $30 \%$, respectivement chez Gladiolus segetum et Gladiolus dubius. Le nombre de racines par pousse est le plus élevé sur ce milieu également : 7,4 et 7,9, respectivement chez Gladiolus segetum et Gladiolus dubius (Figure 3). La 
longueur des racines paraît peu affectée par la concentration de l'AIB et varie de 5,1 à 5,4 cm chez Gladiolus segetum et de 4,6 à 5,0 cm chez Gladiolus dubius.

Tableau 3 : Effet de l’AIB sur l'enracinement in vitro des pousses de Gladiolus segetum et Gladiolus dubius.

\begin{tabular}{|c|c|c|c|c|}
\hline AIB (mg. ${ }^{-1}$ ) & Espèces & $\begin{array}{c}\text { Taux } \\
\text { d'enracinement (\%) }\end{array}$ & $\begin{array}{c}\text { Nombre } \\
\text { des racines }\end{array}$ & $\begin{array}{c}\text { Longueur } \\
\text { des racines (cm) }\end{array}$ \\
\hline 0 & $\begin{array}{c}\text { G.segetum } \\
\text { G.dubius }\end{array}$ & 0 & 0 & 0 \\
\hline 0,1 & G.segetum & 12 & 0 & 0 \\
\hline & G.dubius & 10 & $4,16 \pm 0,4 \mathrm{~b}$ & $5,16 \pm 2,56 \mathrm{a}$ \\
& G.segetum & 26 & $5,2 \pm 0,83 \mathrm{~b}$ & $5 \pm 0 \mathrm{a}$ \\
\hline 0,5 & G.dubius & 24 & $5,33 \pm 3,22 \mathrm{~b}$ & $5,41 \pm 2,87 \mathrm{a}$ \\
& G.segetum & 36 & $7,44 \pm 4,5 \mathrm{a}$ & $5,15 \pm 2,22 \mathrm{a}$ \\
\hline 1 & G.dubius & 30 & $7,92 \pm 3,98 \mathrm{a}$ & $4,57 \pm 2,34 \mathrm{a}$ \\
\hline
\end{tabular}

Les valeurs sur la même colonne portant des lettres distinctes sont significativement différentes $(\mathrm{P}<0,05)$.

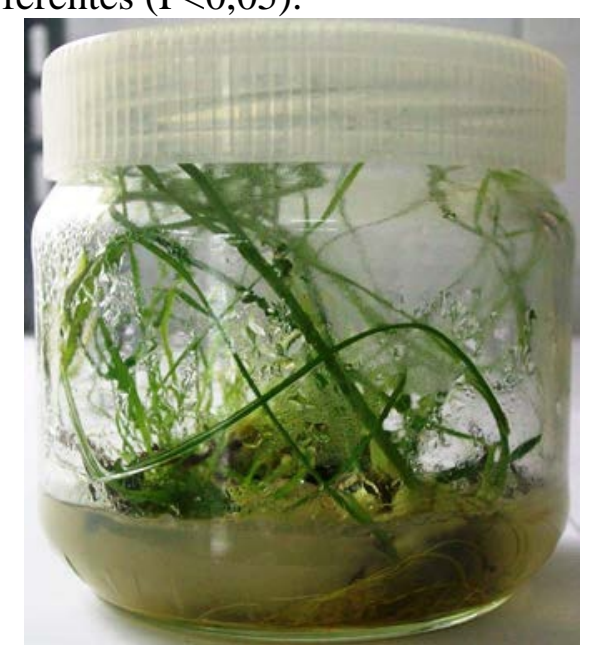

Figure 3 : Enracinement in vitro des pousses de Gladiolus dubius sur le milieu contenant

\section{AIB 1mg. ${ }^{-1}$.}

\section{Phase d'acclimatation}

Les pousses enracinées qui n'ont pas encore formé de cormes ont été transférées en caissettes sur un substrat inerte composé de tourbe et de perlite à proportions égales. Les jeunes plantules maintenues à l'étouffée sous serre, à une température de $27 \pm 3^{\circ} \mathrm{C}$, une humidité relative de $80-85 \%$ ont formé toutes des cormes après 6 semaines de culture. 


\section{Discussion}

Dans la phase d'initiation, les bourgeons apicaux étudiés de deux espèces Gladiolus segetum et Gladiolus dubius n'évoluent que vers le bourgeonnement axillaire sur tous les milieux de culture. En effet, le nombre de pousses le plus élevé a été observé chez le milieu contenant BA $2 \mathrm{mgl}^{-1}$ et AIB 0,5 $\mathrm{mgl}^{-1}$. Ces résultats en accord avec ceux de steinitz et al., (1991) qui montrent que le milieu le plus favorable au bourgeonnement in vitro du glaïeul pour la phase d'initiation doit contenir de BA. D'autre part ce milieu riche en BA, a permis d'avoir 3,83 et 4,1 bourgeons néoformés pour les explants prélevé des bourgeons apicaux, respectivement chez Gladiolus segetum et Gladiolus dubius. Ces résultats sont en accord avec ceux trouvés par Bettaieb (2003), qui a observé le meilleur bourgeonnement de bourgeons apicaux de deux cultivars white friendship et Peter pears de glaïeul sur un milieu pareille à celui sur lequel on a trouvées meilleurs résultats.

Ici on peut relever l'importance de la combinaison hormonale entre l'auxine et la cytockinine (Souyah et al., 2002), puisque la BA seule ne donne pas un nombre élevé de bourgeons malgré son importance dans le bourgeonnement et la régénération (Augé,1989). Les bons résultats obtenus pendant la phase d'initiation sont dut à l'effet conjugué de la présence de benzyljadénine et l'acide indol butyrique (Ben Radhia, 2005). Pour l'enracinement, tous les milieux testés ont abouti à une rhizogenèse des pousses sauf le témoin. L'augmentation de la concentration de l'auxine aboutie à une augmentation de taux d'enracinement ainsi le nombre moyen de racines.la longueur de racine n'a pas affecté par les différentes concentrations d'auxines. La meilleure concentration est celle d'AIB $1 \mathrm{mg} . \mathrm{l}^{-1}$. Ces résultats sont en accord avec ceux de Nhut et al., (2004) qui ont montré que la rhizogenèse des pousses de glaïeul mises en culture in vitro a lieu dans des milieux de culture contenant l'AIB et dont les concentrations varient entre $1 \mathrm{mg} . \mathrm{l}^{-1}$ et $2,5 \mathrm{mg} \cdot \mathrm{l}^{-1}$. Ces résultats sont aussi comparables à ceux trouvés par Dantu et Bhojwani (1995) qui indiquent que le nombre le plus élevé de racines est obtenu en présence de $1 \mathrm{mg}^{-1} \mathrm{l}^{-1} \mathrm{AIB}$ ainsi qu'à ceux de Sinha et Roy (2002) où le meilleur enracinement du Gladiolus primulinus Baker est réalisé en présence de 2 mg. . $^{-1}$ AIB. De Bryn et Ferreir (1992) ont également atteint l'enracinement des pousses cultivées in vitro en utilisant AIB 0,5 mgl-1 et dans un milieu MS/2. L'absence d'une différence pour la longueur de racines pour les différentes concentrations d'auxines peut être expliquée par un effet prévisible, car ce régulateur de croissance est connu pour ces propriétés de stimulation de la rhizogenèse. Le meilleur taux d'enracinement a été observé sur le milieu MS. Ces résultat n’ont pas en accord avec ceux de (De Bryn et Ferreir 1992), qui ont montré que le milieu MS/2 à donnés le meilleure taux d'enracinement et de bulbaison, ceux-ci peut être expliqué par la forte concentration en glucose et fructose interne 
chez les bulbes de Gladiolus sp. Cette concentration interne peut donner un effet irréversible. L'utilisation des différentes concentrations du milieu MS a pour objectif d'assurer un meilleur enracinement des vitropousses issues de la phase de multiplication et de les préparer à la phase d'acclimatation. Pour toutes les phases étudiées il n’y a pas une différence significative entre les deux espèces. Tous les explants provenant de la même région, ceux-ci peut expliquer par l'effet de climat.

\section{Conclusion}

La micropropagation des deux espèces spontanées du genre Gladiolus est possible à partir des différents explants, mais les bourgeons apicaux ont toujours donné les meilleurs résultats. L'initiation nécessitait un milieu contenant l'auxine et la cytochinine, cette combinaison hormonale s'avère nécessaire pour l'initiation et la multiplication des pousses de glaïeul. Le milieu le plus favorable dans cette étude, est celui contenant BA 2 mg..$^{-1}$ et AIB 0,1mg.l $1^{-1}$.

Pour l'enracinement in vitro on a testé différentes concentration hormonales, on a enregistré que le meilleur milieu pour l'enracinement est celui contenant le milieu de base MS et l'AIB $1 \mathrm{mg} \cdot \mathrm{l}^{-1}$.

\section{References:}

Aftab F, Alam M, Afrasiab H. 2008. In vitro shoot multiplication and callus induction in Gladiolus hybridus Hort. Pakistan Journal of Botany 40 (2), 517-522

Ascough G.D., Swart P.A., Finnie J.F., Van Staden J. 2011. Micropropagation of Romulea minutiflora, Sisyrinchium laxum and Tritonia gladiolaris - Iridaceae with ornamental potential. South African Journal of Botany 77, 216-221.

Augé, R., Beauchesne, G., Boccon-Gibod, J., Decourtye, L., Digat, -B., Jalouzot, R.,

Bajaj YPS, Sidhu MMS, Gill APS. 1992. Micropropagation of Gladiolus. In: Bajaj YPS (Eds) Biotechnology in Agriculture and Forestry 20, High-Tech and Micropropagation IV, Springer Verlag, Berlin, 135-143

Begum S. \& Hadiuzzaman S., 1995. In vitro rapid shoot proliferation and corm development in Gladiolus grandiflorus cv. Redbrand. Plant Tissue Cult., 5, 7-11.

Ben Radhia H. 2005. Adaptation to the ornamental horticulture spontaneous gladiolus (Gladiolus segetum Ker Gaul.) in Tunisia. Master of Science, National Agronomic Institute of Tunisia, 65 p.

Bettaieb T., 2003. Régénération in vitro de variants somaclonauux de glaïeul (Gladiolus grandiflorus Hort.) Tolérants aux basses températures. Thèse de doctorat, Institut national agronomique de Tunisie, 136 p. 
Boonvanno K. \& Kanchanapoom K., 2000. In vitro propagation of Gladiolus. Suranaree J. Sci. Technol., 7, 25-29.

Dantų P.K. et Bhojwani S.S., 1995. In vitro corm formation and field evaluation of corm derived plants of Gladiolus. Scientia Hortic. 61,123-128.

De Bryn, M.H. et D.I., Ferreir., 1992. In vitro corm production of Gladiolus dalennii and G. tristis. Plant Cell Tiss. Org. Cult. 31, 123-128.

Edouard LE FLOC'H, Loutfy BOULOS et Errol VELA. 2010. Catalogue synonymique commenté de la FLORE DE TUNISIE. 500p.

Gladiolus tristis. Plant Cell Tissure Organ Cult, 31, 123 - 128.

Goo, D.H., H.Y. Joung and K.W. Kim. 2003. Differentiation of gladiolus plantlets from callus and subsequent flowering. Acta Hort. 620, 339-342.

Grewal HS., Arora JS. et Gosal SS., 1995. Micropropagation of Gladiolus through in vitro cormlets production. Plant Tissue Cult. 5, 27 - 34.

Haouala, F. et Chaieb, E., 2012. Effects of Explant Position and Polarity on Callus Induction and Shoot Regeneration of Gladiolus (Gladiolus hybridus Hort.). Floriculture and Ornamental Biotechnology. 6 (Special Issue 2), 4047.

Hussain I, Mohamed H. \& Rashid Quraishi A., 2001. In vitro multiplication of Gladiolus (Gladiolus crassifolius). Plant Tissue Cult., 11, 121-126.

Hussey G. 1977. In vitro propagation of Gladiolus by precocious axillary shoot formation. Scientia Horticulturae 6, 287-296

Kumar, A., Sood, A., Palni, L.M.S. and Gupta, A.K. 1999. In vitro propagation of gladiolus hybridus hort.: Synergistic effect of heat shock and sucrose on morphogenesis. Plant Cell Tissue Org. Cult. 57,105-112.

Margara J. 1984. Néoformation de méristèmes et embryogenèse somatique. In: INRA (Eds) Bases de la Multiplication Végétative. Les Méristèmes et l'Organogenèse, Paris, 75-175

Murashige T. \& Skoog F.A., 1962. A revised medium for rapid growth and bioassays with tobacco tissue culture. Physiol. Plant., 15, 473 - 492.

Nagaraju V. \& Parthasarathy V.A., 1995. Effect of growth regulators on in vitro shoots of Gladiolus hybridus. Folia Horticulturae, 7(2), 93-100.

Nhut DT, Teixeira da Silva JA, Huyen PX, Paek KY.2004. The importanceof explant source on regeneration and micropropagation of Gladiolus by liquid shake culture. Scientia Horticulturae 102, 407-414

Prasad, V.S.S. and S.D. Gupta. 2006. In vitro shoot regeneration of gladiolus in semi-solid agar versus liquid cultures with support systems. Plant Cell Tiss. Org. Cult. 87,263-271

Priyakumari, I. and V.L. Sheela. 2005. Micropropagation of gladiolus cV. 'Peach Blossom'

through enhanced release of axillary buds. J. Trop.Agri. 43(1-2),47-50.

Roy S., Gangopadhyay G., Bandyopadhyay T., Binoy K., Datta S., Kalyan K. et Mukherjee., 2006. Enhancement of in vitro micro corm production in 
Gladiolus using alternative matrix. African Journal of Biotechnology. 5 (12). 1204-1209.

Salhi I., 2007. Contribution to the study of salinity tolerance in gladiolus (Gladiolus grandiflorus Hort.) cultured in vitro and in vivo. Master of Science. higher agricultural institute of Chott Meriem, $60 \mathrm{p}$.

Sen J, Sen S. 1995. Two-step bud culture technique for a high frequency regeneration of Gladiolus corms. Scientia Horticulturae 64, 133-138

Sinha P. \& Roy S.K., 2002. Plant regeneration through in vitro cormel formation from callus culture of Gladiolus primulinus Baker. Plant Tissue Cult., 12,( 2), 139-145.

Souyah, N.,Khouja, M.L., Rejeb, M.N et Bouzid, S., 2002. Micropropagation of a shrub sylvopastoral, Atriplex halimus L. (Chénopodiacées). Faculty of Sciences of Tunis. p 131 - 135.?????????

Steinitz B, Cohen A, Goldberg Z \& Kochba M. 1991. Precocious gladiolus corm formation in liquid shake cultures. Plant Cell Tiss. Org. Cult. 26,63-70

Ziv M. \& Lilien-Kipnis H., 2000. Bud regeneration from inflorescence explant for rapid propagation of geophytes In vitro. Plant Cell Rep., 19, 845850.

Ziv M. 1979. Transplanting Gladiolus plants propagated in vitro. Scientia Hort. 11,257-260 